\title{
Twin universes: Universal laws of thermodynamics
}

\author{
Leong Ying \\ Thermo Fisher Scientific, Hoboken, USA
}

Email address:

leong.ying@thermofisher.com, klystar@gmail.com

\section{To cite this article:}

Leong Ying. Twin Universes: Universal Laws of Thermodynamics. American Journal of Modern Physics. Special Issue: New Science Light Path on Cosmological Dark Matters. Vol. 4, No. 1-1, 2015, pp. 1-4. doi: 10.11648/j.ajmp.s.2015040101.11

\begin{abstract}
Big Bang is the widely accepted model of the beginning of our cosmos some 13.7 billion years ago from a single point source into its present-day expansion which is continuing at an unexplained accelerating rate. All known physical processes in our universe are governed by the laws of thermodynamics, except for the moment of creation which defies the conservation of energy even though the cosmos is the most ideal isolated system. Other observable anomalies that this single 4-dimensional space-time universal existence cannot account for include missing antimatter, rapidly rotating galaxies, faster-than-light-speed quantum entanglement and proton stability. Applying a universal conservation laws to both the fundamental quantities of energy and entropy forces the beginning of our cosmos to divide into a zero-balanced twin universes existence. This fully conserved 10-dimensional cosmos is now able to adequately explain these anomalies with model calculations that match the experimental data from cosmology and high-energy physics.
\end{abstract}

Keywords: Twin Universes, Thermodynamics, Dark Energy, Dark Matter, Antimatter, Quantum Entanglement

\section{Introduction}

The first science model to interpret the constituents of Dark Energy and produce a numeric calculation of the accelerating value of Hubble's constant was based on a proposed Twin Universe theory [1]. This cosmic existence comes about by forcing the fundamental quantities of energy and entropy to be conserved at the moment of the Big Bang singularity point of creation. To maintain zero-balanced conservation laws of thermodynamics, two parallel universes must coexist with equal but opposite values. Parameterization of dual positive and negative quantities can be formulated in terms of Santilli's isodual mathematics [2].

There are many proposed models of multiverses (from two to infinite universes) such as Ellis [3]. Sakharov [4] proposed the breaking of charge-parity $(C P)$ symmetry led to the baryonic imbalance that created a surplus of matter in our present-day universe. The imbalance would only need to be exceptionally small of the order of 1 part in $10^{10}$ at the moment of creation. Petit [5] used the same concept in his cosmological model whereby two universes were born from the same initial Big Bang singularity. The twin universe with its reverse arrow of time $(T)$ would be mainly populated by antimatter. As a whole the $C P T$-symmetry would maintain global un-violated parity between the combined universes.

\section{Universal Laws of Thermodynamics}

The laws of thermodynamics are the basic foundation of physical principles that governs all of nature. There are no fundamental mathematical formulations for these rules; the laws are purely derived from measureable observations. The first law of thermodynamics mandates conservation of energy, and states that heat is a form of energy. The current second law of thermodynamics states that the entropy of an isolated macroscopic system never decreases. Life as we know it could not exist if these rules were violated. However, present-day observations in cosmology and high-energy physics appears not to conform with current standard models of our universe, and therefore a new universal laws of thermodynamics is proposed to shift the paradigm back to match the measureable observations.

\subsection{First Universal Law of Thermodynamics}

The first law of thermodynamics relating to the conservation of energy is still valid within the new universal definition.

\subsection{Second Universal Law of Thermodynamics}

The new proposed second universal law of thermodynamics 
now mandates that entropy is also conserved as with the first law.

\subsection{Universal Postulates}

To define how the new proposed universal laws of thermodynamics can operate in our observed nature, five postulates are cited.

\subsubsection{First Universal Postulate}

This states that the Total Universe is composed of two anti-symmetric systems, a Positive Universe and a Negative Universe. The two universes have identical 4-dimensional space-time coexisting on opposite sides of a 2-dimensional membrane, giving a total of 10-dimensions for the combined cosmos.

\subsubsection{Second Universal Postulate}

This states that energy (E) can flow between both universal systems, but the total energy in the Total Universe must be conserved.

$$
\mathrm{E}_{\text {Total }}=\mathrm{E}_{\text {Positive }}+\mathrm{E}_{\text {Negative }}
$$

Before the beginning of creation, there was no presence of energy $\left(E_{\text {Total }}=0\right)$, so to conserve energy at the point of creation and thereafter in obedience to the first universal law of thermodynamics, the cosmos divides into parallel twin universes.

$$
\mathrm{E}_{\text {Positive }}=-\mathrm{E}_{\text {Negative }}
$$

This zero-balanced universal conservation of energy requires that all energy-mass states must have identical quantities but with reversed polarity of values.

\subsubsection{Third Universal Postulate}

This states that entropy (S) can flow between both universal systems, but the total entropy in the Total Universe must be conserved.

$$
\mathrm{S}_{\text {Total }}=\mathrm{S}_{\text {Positive }}+\mathrm{S}_{\text {Negative }}
$$

To conserve entropy at the point of creation in obedience to the second universal law of thermodynamics:

$$
\mathrm{S}_{\text {Positive }}=-\mathrm{S}_{\text {Negative }}
$$

This zero-balanced universal conservation of entropy requires that all processes and actions must be repeated but acting in reversed sense of direction. So for a photon that behaves as a particle on one side of the universal membrane then its identical twin photon behaves simultaneously with wave properties on the opposite cosmic frame of reference.

\subsubsection{Fourth Universal Postulate}

This states that gravity dictates the direction of entropy change (time). Time is a consequence of the change of entropy of a system. If nothing changes, then time would stand still. Positive increasing entropy leads to a forward motion of time. For example, a cup falls off a table and smashes into multiple fragments, which in common terms we state that the system (cup) increases with positive entropy from a single state (whole cup) to multiple states (fragments of cup) in the forward direction of time. In real experience, the broken fragments never forms back into a single whole cup and jump back onto the table, that is, time never reverses in real life. In the parallel universe negative increasing entropy leads to a backward motion of time. However, since all quantities are negative including energy, leading to repulsive gravity of all matter, then mass as we define it would never condense into an equivalent solid cup in this parallel existence.

\subsubsection{Fifth Universal Postulate}

This states that life on Earth exists only in the Positive Universe. Negative energy leads to repulsive gravity of mass and negative entropy leads to a reversed duality of existence, hence a physical observer can only perform physical experiments in the Positive Universe, where increasing entropy is the normal approaching state of equilibrium, and the original second law of thermodynamics is therefore not violated for such an observer.

\section{Experimental Observables}

For any new scientific theories or models to be validated, it must be able to make correct predictions and produce calculable values that are consistent with research data. The Twin Universe model stemming from the proposed Universal Laws of Thermodynamics will be compared to current experimental observables to check for its potential validity.

\subsection{Dark Energy}

The Wilkinson Microwave Anisotropy Probe [6] has determined the Hubble's constant $(\mathrm{H})$ at $71 \mathrm{~km} \cdot \mathrm{s}^{-1} \cdot \mathrm{Mpc}^{-1}$. Hubble's law [7] is considered the first observational basis for an expanding universe and supporting evidence of the Big Bang model. Dark Energy is the unknown constituent that propels the present accelerating state of our cosmos. From a Twin Universe perspective, it is proposed that stellar nuclear fusion replicated in the reverse mirrored universe can account for the negative-energy pressure (Dark Energy) that drives our present-day accelerated cosmic expansion. In our positive quantified side of the universe, nuclear fusion within stars emits radiation as energy waves. In the mirror twinned universe all processes are duplicated but with reversed negative polarity. Hence in the negative quantified side of the universe, the identical fusion processes will emit radiation as individual particle energy photons that experience gravitational repulsion. Formulated by Ying [8] for point masses $(\mathrm{m})$ condensing on the 2-dimensional membrane with an area mass density $\rho=m / \pi r^{2}$, where $r$ is the spatial distance between the point masses, the repulsive gravitational force moving at the speed of light (c) will stretch the membrane at an expanding rate $\left(\mathrm{U}_{\mathrm{r}}\right)$ given by the following equation:

$$
\mathrm{U}_{\mathrm{r}}=\pi \mathrm{G} \rho / \mathrm{c}
$$

This simple model calculates based on the energy released from the nuclear fusion processes in standard stars an 
estimated cosmic expansion rate $\mathrm{U}_{\mathrm{r}} \sim 160 \mathrm{~km} \cdot \mathrm{s}^{-1} \cdot \mathrm{Mpc}^{-1}$. The computed value can be more accurately determined by refining the model to be more representative of the distribution of the stars and the emitted energy particle waves. Since both universes exist on the opposing surfaces of the membrane they will therefore share the same experience of a stretching membrane.

\subsection{Dark Matter}

The measured rotational velocities of galaxies are too great to maintain stability for the observable mass within each galaxy [9]. Dark Matter was added to compensate for this apparent lack of galactic mass. However, there are proponents that claim both Dark Energy and Dark Matter are the same components referred to as Dark Fluid [10]. The repulsive gravitational expansion in the intermediate vacuum of space created by the condensing stellar nuclear fusion in the mirror twinned universe is also generating the compressive force on the galactic cluster of stars. Current experimental estimates of the percentage of Dark Matter is 23\% with Dark Energy around $73 \%$, and the remaining $4 \%$ of normal baryonic matter. If indeed the differing effects are producing a double-counting of the same unobserved Dark Fluid, then the actual percentage matches the required $50 \%$ based on a Twin Universe model whereby the negative energy-mass constitute the opposing Dark Fluid.

\subsection{Antimatter}

Rowlands [11] have proposed the existence of virtual-particles that are duplicates of normal matter and antimatter. This four distinct particle states is also a consequence of a Twin Universe model where positive matter and antimatter have identical but reversed negative twinned particles.

The true meaning of time is still hotly debated as to whether it is merely a mathematical concept or a real fundamental quantity of the universe. Newton [12] theorized that time passes by independent of other interactions with the rest of the universe, whereas Einstein [13] special theory of relativity states that time is not absolute and is dependent on the relative motion of the observer. Ying [14] postulate that time is a measureable physical quantity and associated with entropy in the same nature that space is associated with matter (energy). Table 1 lists the proposed physical properties of space-time based on a Twin Universe viewpoint.

Table 1. Physical space-time of twin universes

\begin{tabular}{ll}
\hline Space & Time \\
\hline Fabric of energy & Fabric of entropy \\
Energy is static capacity to work & Entropy is dynamic process to work \\
There are multiple degrees of energy and multiple dimensions of space & There is single degree of entropy and single projection of time \\
There are two directions of energy-space (positive and negative) & There are two directions of entropy-time (positive and negative) \\
Increasing positive energy curves space inward & Increasing positive entropy moves time forward \\
Decreasing negative energy curves space outward & Decreasing negative entropy moves time backward \\
\hline
\end{tabular}

The total cosmos can be treated as expanding twin universes where there existed a unitary super-particle and super-antiparticle at the origin of the Big Bang event. The initial mixing of these super-states between the two orthogonal universes provides a numeric surplus of matter over antimatter that is consistent with observed measurements within our universe of $10^{9}$ [15].

\subsection{Proton Stability}

One major problem in physics is the predominance of matter over antimatter in our universe, which suggests that the universe as a whole has nonzero positive baryonic number density. This apparent non-conserving baryonic number would in principle cause protons to decay, which has never been experimentally observed. In a Twin Universe model the ratio of matter to antimatter is perfectly balanced by its reverse quantized twinned particle states, and therefore in this super-symmetric system the baryonic (quark) number is conserved.

\subsection{Quantum Entanglement}

Entanglement is the unexplained quantum phenomenon in which two vastly separated particles share the same existence, and thereby apparently defying the speed-of-light limitation when they interact simultaneously. In quantum mechanics both particles are described by a single wave function. In a Twin Universe model the zero-balanced conservation of energy forces all matter and antimatter particles to have duplicated twinned particles existing in an opposing state of duality. And the consequence of zero-balanced conservation of entropy forces these twinned particles to be inherently linked in their interactions through superposition of their wave states.

\section{Conclusion}

Table 2 summarizes the difference in interpretation of current standard models of the cosmos and that based on a Twin Universe existence. 
Table 2. Observed properties of the cosmos

\begin{tabular}{|c|c|c|}
\hline Properties & Current Scientific Models & Twin Universe Model \\
\hline Laws of thermodynamics & Cosmos violates energy conservation law & Cosmos obeys conservation laws \\
\hline Universal expansion & Dark Energy & $\begin{array}{l}\text { Stellar nuclear fusion with repulsive gravity stretches } \\
\text { common membrane }\end{array}$ \\
\hline Galactic rotation & Dark Matter & $\begin{array}{l}\text { Stellar nuclear fusion with repulsive gravity compresses } \\
\text { galactic star clusters }\end{array}$ \\
\hline Matter to antimatter ratio & Unexplained & $\begin{array}{l}\text { Orthogonal mixing of unitary super-particles at point of } \\
\text { creation }\end{array}$ \\
\hline Proton stability & Decay due to unbalanced baryonic number & Stable due to conserved baryonic number \\
\hline Quantum entanglement & Violates speed of light & $\begin{array}{l}\text { Interactions through superposition of twinned particle wave } \\
\text { states }\end{array}$ \\
\hline Space-time dimensions & Four (3 space, 1 time) & Ten (8 space, 2 time $)$ \\
\hline
\end{tabular}

\section{References}

[1] L. Ying, Nuclear fusion drives cosmic expansion, vol. 137. Springer Proceedings in Physics: Springer, 2011, pp.379-381.

[2] R. M. Santilli, Isodual theory of antimatter, Fundamental Theories of Physics: Springer, 2006.

[3] G. F. R. Ellis, U. Kirchner and W. R. Stoeger, Multiverses and physical cosmology, vol. 347, Royal Astronomical Society, 2004, pp. 921-936.

[4] A. D. Sakharov, Violation of CP symmetry, C-asymmetry and baryon asymmetry of the universe, vol. 5, JETP Letter, 1967, pp. 24-27.

[5] J. P. Petit, Enantiomorphic universes with opposite time arrows, vol. 263, Comptes rendus de l'Academie des Sciences, 1977, pp. $1315-1318$.

[6] C. L. Bennett, M. Bay, M. Halpern, et al, The microwave anisotropy probe mission, vol. 583, The Astrophysical Journal, 2003, pp. 1-23.

[7] E. Hubble, A relation between distance and radial velocity among extra-galactic nebulae, vol. 15, Proceedings of the National Academy of Science USA, 1929, pp.168-173.

[8] L. Ying, Dark Energy is stellar nuclear fusion replicated in a mirrored universe, vol. 4, The Open Astronomy Journal, 2011, pp. 54-56.

[9] V. C. Rubin and W. K. Ford, Rotation of the Andromeda nebula from a spectroscopic survey of emission regions, vol. 159, The Astrophysical Journal, 1970, pp. 379-403.

[10] H. S. Zhao, An ecological approach to problems of dark energy, dark matter, MOND and neutrinos, vol. 140, Journal of Physics Conference Series, 2008, pp. 1-12.

[11] P. Rowlands, Zero to infinity, World Scientific Publishing, 2007.

[12] I. Newton, Philosophiae Naturalis Principia Mathematica, 1687.

[13] A. Einstein, Zur elektrodynamik bewegter korper, 1905.

[14] L. Ying, Anti-antimatter, vol. 13, Journal of Computational Methods in Sciences and Engineering, 2013, pp. 303-305.

[15] L. Ying, Pi is fundamental cosmic frequency in expanding universe, vol. 6, The Open Astronomy Journal, 2013, pp. 7-9. 